International Journal of Engineering \& Technology, $7(4.5)(2018) 134-137$
International Journal of Engineering \& Technology
SPC
Website: $w$ ww.sciencepubco.com/index.php/IJET
Research paper

\title{
Developments for Diabetic Retinopathy Detection and Classification: A Review
}

\author{
Pooja M. Pawar ${ }^{1 *}$, Avinash J. Agrawal ${ }^{2}$ \\ ${ }^{1,2}$ Shri Ramdeobaba College Of Engineering and Management, \\ Nagpur, Maharashtra, India. \\ *Corresponding author E-mail: 1* pooja27pawar@gmail.com, 2agrawalaj@rknec.edu
}

\begin{abstract}
Diabetes is characterized by impaired metabolism of glucose caused by insulin deficiency. Diabetic retinopathy is the eye disease, is caused by retinal damage which is generally formed as a result of diabetes mellitus. It is a serious vascular disorder for which early detection and the treatment are required to inhibit the intense vision loss. Also, the diagnosis entails skilled professionals for detection because non-automatic screening methods are very time consuming and are not that efficient for a large number of retinal images. This paper provides a broad review of various techniques and methodologies used by the authors for diabetic retinopathy detection and classification. Furthermore, most recent work and developments are studied in this paper. We are proposing an advanced deep learning CNN approach for automatic diagnosis of DR from color fundus images.
\end{abstract}

Keywords: CNN; deep learning; diabetic retinopathy; fundus images; retina.

\section{Introduction}

Retina is the complex organ of a human eye. It is the third and inner layer of the eye and the only part of the brain which is reached by daylight. Its purpose is to convert the light which has been focused by the lens into electrical signals and then send them back to the brain where the essential visual cortex waits to process those signals further for visual recognition. Diabetes Mellitus occurs when too much sugar is circulating in the human blood stream. Diabetic Retinopathy or DR is commonly called as Diabetic Mellitus which demonstrates itself in the retina. The symptoms of DR are not that prominent and hence patients having this disease are not concerned about the changes in vision. DR is the principal origin of complete vision loss. According to the International Diabetes Federation in 2015, about 415 million grown-ups had diabetes throughout the world and they projected this figure to grow by $50 \%$ to 642 million by 2040 . Advanced treatment and diagnosis of DR may reduce the possibility of critical vision loss or blindness.

Retinal images usually contain various elements like macula, optic disk (called as OD), blood vessels, fovea etc. Clinicians generally categorize DR into two main classes known as proliferative DR(PDR) and Non-Proliferative (NPDR) which is the primary stage of DR. Microaneurysms are minor areas of swelling in the retina's blood vessels which appear as red dots that occur due to lack of oxygen. Yellow lipids caused by the damage of blood vessels and emerged as bright yellow lesions are called as exudates. Exudates have two types that are Hard exudates and Soft exudates which are named as Cotton Wool Spots. Hemorrhages are big blood clots caused by ischemia in the retina. NPDR has three classes mild, moderate and severe DR. Mild DR contains at the minimum one Microaneurysm which exists in the retina and it is the earliest clinically noticeable lesion, it also contains some hard or soft exudates. Moderate DR includes some Microaneurysms or dot hemorrhages as well as Soft exudates and IRMA. In Severe DR, the retina has various microaneurysms and intraretinal hemorrhages in all the four quadrants.

The modern attempts in the field of diabetic retinopathy include the extraction of lesions such as microaneurysms, blood vessels and exudates etc through which the classification is performed. The objective of this research paper is to encapsulate the recent research developments which have been performed for automatic diagnosis of DR detection and monitoring system. The image processing methods already achieved precise results in DR detection and it is the active research area at the current stage. Also, this paper proposes the deep learning model for the recognition of diabetic retinopathy which automatically learns features by using convolutional neural network i.e. $\mathrm{CNN}$.

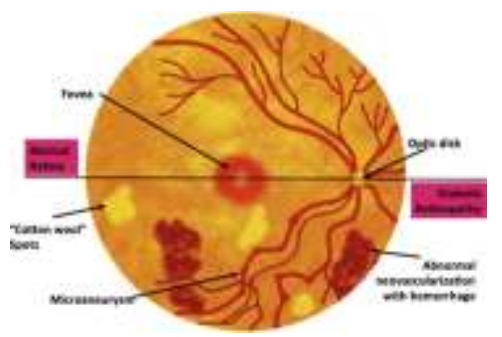

Fig. 1: Retinal image and its features

\section{Related Work}

This section covers various methods and procedures adapted for DR screening. G. G. Gardner et al. [1] deftly presented artificial neural network and some pre-processing techniques for diabetic retinopathy screening. They applied back-propogation neural network for analyzing the retinal fundus images of 147 patients with DR and 32 from normal subjects. They implemented exudates 
detection by using maximum median filtering method, in this method the center pixel value is swapped by the maximum value and similarly, for hemorrhages they used minimum median filtering. Later, Nine(9) point averaging filter along with the Sobel edge detection operator was used for blood vessel detection. They achieved $88.4 \%$ sensitivity and $83.5 \%$ specificity for Yes or No binary classification using the neural network.

R.Priya and P. Aruna [2] proficiently addressed this problem by using SVM, PNN \& Bayesian Classification models. The input images first pre-processed by converting RGB to Grayscale image and adaptive histogram equalization for contrast improvement in the image, later they applied a discrete wavelet transform for reducing the size of the converted image followed by a matched filter and fuzzy C-means segmentation. Feature extraction was obtained by using Green channel extraction, thresholding, and morphological operations. Among SVM, PNN and Bayesian Classification, SVM was found more efficient with an accuracy of $97.6 \%$, Bayes Classifier with $94.4 \%$ and PNN with $89.6 \%$.

Sohini Roychowdhury, K.Parhi et al. [3] brilliantly proposed an important contribution by introducing a DREAM system in which a novel two-stage hierarchical classification algorithm was used for automatic recognition and classification. In primary step, a minimum-intensity maximum solidity algorithm used to detect the regions corresponding to OD and vasculature. In the second stage, lesion classification was implemented on the basis of a red and bright lesion in the following two phases: In phase-1, lesions are classified as true and non lesions and in phase-2, red lesions identified as micro-aneurysms and hemorrhages. For lesion classification, the authors used GMM, SVM, KNN and AdaBoost methods This DREAM system achieved $90.4 \%$ accuracy.

M.Rajesh Babu et al. [4] proposed a method for exudates classification from color fundus images. First, pre-processing was done by using color space conversion and histogram equalization was used for Contrast Enhancement. Optic disc identified by using Edge detection technique and Dilation operation was used for Blood vessels removal because they have a similar concentration level of exudates. Finally, exudates were identified using Closing operation. Later for classification, the segmented image of exudate was delivered to an SVM classifier. They used Gray Level Cooccurrence Matrix (GLCM) for extraction of input features for SVM.

Jaydeep De et al. [6] introduced the graph theory mechanism to uncover the filamentary irregularities in the retinal fundus image, it is implemented for finding the filamentary arrangement and then disconnect the thread structure into disjoint of a thread. The implementation has been done in two steps, segmentation and tracing. The outcome of segmentation is then translated into 1-pixel skeleton map (i.e. junction, body, terminal, and root) thickness and assembled the digraph from the converted skeleton map. The theorem called matrix-forest was applied for label propogation and has been used for tracing outcome on the digraph.

\section{Image Datasets used for DR Screening and Research}

The Fundus camera is a device which is used to capture the retinal image. The selection of dataset to train and test the data plays an important role in the judgment of any proposed systems. Various standard datasets are publicly available for the assessment of algorithms introduced for the computerized screening and diagnosis of DR. There are seven databases which are available in public domain such as DRIVE, STARE, DIARETDB, e-ophtha, HEIMED, Retinopathy Online Challenge (ROC), and Messidor.

The DRIVE (called Digital Retinal Images for Vessel Extraction) dataset, here the images were acquired in The Netherlands. The images were obtained by using a Canon CR5 non-mydriatic 3CCD camera with a 45-degree field of view (FOV). It consists of 40 color fundus images, which are stored in the TIFF format. STARE is the STructured Analysis of the Retina database involves a complete set of 400 raw images applied for blood vessel segmentation.
Another is MESSIDOR, a widespread dataset used for DR screening contains 1200 eye images. The images were obtained by using three different ophthalmologic departments using color video 3 CCD camera with a 45- degree field of view (FoV).

DIARETDB1 and DIARETDB0 contain images which are 1500x1152 pixels wide and stored in PNG format. It has normal and abnormal images containing symptoms of retinopathy.

\section{Performance and Discussion}

For analyzing the achievement of all the methods discussed in the related work section, authors considered some quality parameters such as sensitivity, specificity, and accuracy. These parameters are calculated as:

Accuracy $=$ Total $(\mathrm{TP}+\mathrm{TN}) /$ Total number of individuals in population

Sensitivity $=$ True Positive $/($ True Positive + False Negative $)$

Specificity $=$ True Negative $/($ True Negative + False Positive $)$.

Accuracy is usually called as the Area Under the Curve (AUC). The methods discussed above for the detection of diabetic retinopathy, all used different dataset and used various image processing and machine learning algorithms used for extracting the features of retinal fundus images and classification as well. The whole discussion is summarized in Table 1.

In this research paper, we have presented various previous works done to implement the image screening and procedures to detect the stages of DR. Majority of the previous work required manual methods for extracting the features from given images before being input to an SVM classifier. Manual methods are sometimes time consuming if the dataset contains large number of images. Recent developments got significant benefits by making use of machine learning and computer vision techniques but there are still some problems with accuracy. If we consider Support Vector Machine (SVM) classifier, it is a time-consuming process for training the model if used for large datasets. Also, SVM does not provide an unambiguous result if our dataset contains noise. The problem with KNN (K-Nearest Neighbors) is that it generally gives a non-zero distance for the images of the same subject with same orientation and lighting conditions with different locations. Also, Bayes classifier assigns zero (0) probability if the particular variable in the testing dataset is not observed in the training set and generally not able to make predictions.

Authors achieved great results with the use of image processing and machine learning concepts along with computer vision but much work demanded feature extraction from the input images before fed to the classifier. Here this paper is proposing a method in which we are using Deep learning mechanism to train the classifier. We are aiming to reduce the required time to complete the whole procedure by using convolutional neural networks which require less training time corresponds to the above methods like SVM, ANN and Bays classifiers etc. CNN has the ReLu activation function which is called as Rectified Linear Unit, it is better than the sigmoid and tanh function because it is more efficient and the gradient never saturates in the positive region. 
Table 1: Result of various DR detection and classification techniques

\begin{tabular}{|c|c|c|c|c|c|c|}
\hline \multirow{2}{*}{$\begin{array}{l}\text { Sr. } \\
\text { No. }\end{array}$} & \multirow[t]{2}{*}{ Authors } & \multirow[t]{2}{*}{ Datasets } & \multirow[t]{2}{*}{ Techniques } & \multicolumn{3}{|c|}{ Evaluation Results } \\
\hline & & & & $\begin{array}{c}\text { Sensitivity } \\
(\%)\end{array}$ & Specificity (\%) & Accuracy \\
\hline 1 & G G Gardner et al.[1] & $\begin{array}{c}\text { Clinical data } \\
\text { (Fundus images) }\end{array}$ & $\begin{array}{c}\text { Artificial Neural Net- } \\
\text { works }\end{array}$ & 88.4 & 83.5 & $\begin{array}{l}93.1 \% \text { for exudates } \\
\text { and } 73.8 \% \text { for hemor- } \\
\text { rhages detection }\end{array}$ \\
\hline 2 & R.Priya and P. Aruna [2] & DIARETDB0 & $\begin{array}{l}\text { SVM, PNN \& Bayesian } \\
\text { Classification models }\end{array}$ & $\begin{array}{l}\text { SVM: } 96.36 \\
\text { PNN: } 90.90 \\
\text { Bayes: } 92.72\end{array}$ & $\begin{array}{l}\text { SVM: } 90.00 \\
\text { PNN: } 70.00 \\
\text { Bayes: } 80.00\end{array}$ & $\begin{array}{l}\text { SVM: } 95.38 \% \\
\text { PNN: } 87.69 \% \\
\text { Bayes: } 90.76 \%\end{array}$ \\
\hline 3 & $\begin{array}{c}\text { Sohini Roychowdhury, Parhi } \\
\text { et al. } \text { [3] }\end{array}$ & Messidor & $\begin{array}{c}\text { GMM, SVM, KNN, and } \\
\text { AdaBoost }\end{array}$ & 100 & 53.16 & 0.904 \\
\hline 4 & Jaydeep De et al. [6] & $\begin{array}{l}\text { DRIVE and } \\
\text { STARE }\end{array}$ & $\begin{array}{c}\text { Graph Theoretical Ap- } \\
\text { proach }\end{array}$ & - & - & 0.546 \\
\hline
\end{tabular}

\section{Proposed Work}

Machine learning has gained most of the attention in the recent state-of-art technology developments. It has many image processing uses such as biomedical imaging, satellite image processing, Artificial Intelligence such as object detection. Deep learning which is the subpart of machine learning has recently gained accurate results in many healthcare systems like skin cancer diagnosis, brain tumor detection, breast cancer identification and diabetic retinopathy recognition etc.

Convolutional Neural Networks (CNN), a class of DL, has a spectacular record for applications in digital image analysis and interpretation, along with medical imaging and video analysis. It is also used in Natural Language Processing (NLP) and drug discovery etc. The flowchart of our proposed diabetic retinopathy classification model is represented in Fig. 2.

It contains following building blocks:

\subsection{Data Augmentation}

The input fundus images are first sent to data augmentation block. The neural network usually demands a large number of training data or images to achieve better results in deep learning. Image augmentation is the addition of new artificial data obtained from the given set of images which can be used for better prediction. This can be done by using various methods of processing such as random cropping, vertical mirroring will give us different examples of images in the training set, rotations, color shifting, shearing, and flips, etc.

\subsection{Preprocessing}

The dataset often contains images from patients of different age groups and different levels of brightness in fundus photography. This affects the intensity values of a pixel within the images hence we need preprocessing like normalization, gray scale conversion etc. The simple and initial preprocessing step is zero-center the data followed by normalization.
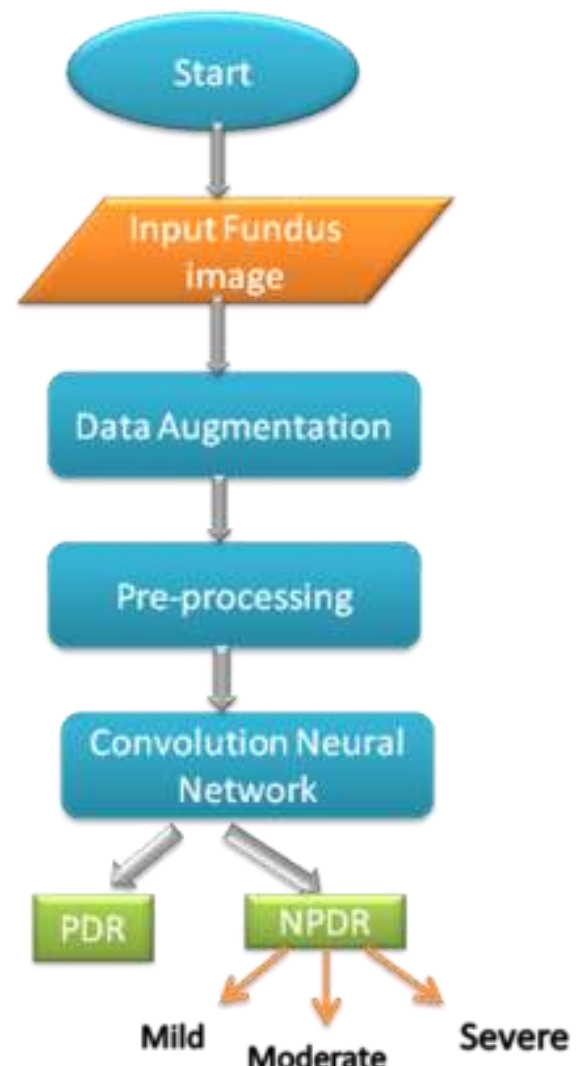

Fig. 2: Flowchart of proposed model

\subsection{Convolutional Neural Network}

CNN contains following set of layers for classification:

\subsubsection{Convolutional Layer}

The Convolutional layer is an important component of CNN which performs most of the computational task. The initial goal of convolution in case of a $\mathrm{CNN}$ is to extract features from the input image. Here we define a weight matrix whose function is to get features from the images. It acts as a filter in the given image which is used to extract particular data from the original matrix. Convolution conserves the orientation in space between pixels by learning the features of an image using small squares of input data.

\subsubsection{Pooling layer}

Whenever the given images are very large, it is required to reduce the number of trainable elements. Spatial Pooling also called subsampling or downsampling. Pooling is implemented mainly for reducing the dimensional size of the image. Maxpooling is a common form of pooling.

Here, we define a spatial neighborhood and take the greatest element from the rectified feature map within the particular window. 


\subsubsection{ReLU layer}

ReLU is named as Rectified Linear Unit and is a nonlinear operation. Its function is given by:

Output $=$ Max (zero, Input)

It is the most frequently used activation function. This is non linear operation as the errors can be evenly backpropagated in this and it has many layers of neurons which are being activated by using the ReLU function.

\subsubsection{Dropout Layer}

It is known as the regularization technique and is used to prevent the network over-fitting. Its goal is to produce improved results for neural networks in supervised learning operations. During the training process, some neurons in this layer are dropped in a random fashion.

\subsubsection{Fully Connected Layer}

It is the conventional Multi-Layer Perceptron which makes use of a special softmax activation function in the final layer. It is named as "Fully Connected" because each neuron in the preceding layer is given as an input to every neuron on the next layer. Each pixel is interpreted as a distinct neuron in this layer. The number of neurons in this layer is equal to the number of classes to be predicted.

\subsubsection{Classification Layer}

It is the final layer. This is the softmax layer used to categorize the input fundus retinal images used single-class or multiclass classification.

\section{Conclusion and Future Work}

We have elaborated the previous and recent work in the domain of diabetic retinopathy which emphasizes the methodologies and techniques required to detect the disease among patients. Though significant implementation has been accomplished in this field, there are many areas to work in this domain like vessel segmentation and detection of arteries and veins etc. We also proposed our deep learning technique for identification and grading of DR from fundus images using the recent $\mathrm{CNN}$ model.

\section{References}

[1] G.Gardner, D.Keating, T.H.Willamson, A.T.Elliott, "Automatic detection of diabetic retinopathy using an artificial neural network: a screening Tool", Brithish Journal of Opthalmology,(1996);80:940-944

[2] R.Priya, P.Aruna, "Diagnosis of diabetic retinopathy using machine learning techniques", ICTACT Journal On Soft Computing, July 2013, Volume: 03, Issue: 04.

[3] S.Roychowdhury, D.D.Koozekanani, Keshab K.Parhi, "DREAM: Diabetic Retinopathy Analysis Using Machine Learning", IEEE Journal of BioMedical and Health Informatics, Vol.18, No 5, September (2014).

[4] M.Rajesh Babu, BVNR Siva Kumar, P.Rakesh Kumar, "Diagnosis of Diabetic Retinopathy Using Morphological Process and SVM Classifier", September 2016 | IJIRT | Vol. 3, Issue 4 | ISSN: 23496002.

[5] Mahendran Gandhi, Dr. R. Dhanasekaran, "Diagnosis of Diabetic Retinopathy Using Morphological Process and SVM Classifier" IEEE International conference on Communication and Signal Processing, April 3-5, 2013, India.

[6] Jaydeep De, Li Cheng, Xiaowei Zhang, Feng Lin, Huiqi Li, Kok Haur Ong, Weimiao Yu, Yuanhong Yu, and Sohail Ahmed, "A Graph-Theoretical Approach for Tracing Filamentary Structures in Neuronal and Retinal Images", IEEE Transactions On Medical Imaging, Vol. 35, No. 1, January 2016.
[7] Michael D. Abràmoff, Mona K. Garvin, Milan Sonka, "Retinal Imaging and Image Analysis", IEEE Reviews in biomedical engineering, vol. 3, 2010

[8] Nair, V., Hinton, G.E.."Rectified linear units improve restricted boltzmann machines". In: Proceedings of the 27th International Conferenceon Machine Learning (ICML-10). 2010, p. 807-814.

[9] Nitish Srivastava, Geoffrey Hinton, Alex Krizhevsky, Ilya Sutskever,Ruslan Salakhutdinov. "Dropout: A Simple Way to Prevent NeuralNetworks from Overfitting". Journal of Machine Learning Research15 (2014).

[10] J. Nayak, P. Bhat, U. R. Acharya, C. M. Lim, M. Kagathi, "Automated identification of diabetic retinopathy stages using digital fundus images,” J. Med. Syst., vol. 32, 2008, pp. 107-115.

[11] M.R.K. Mookiah, U. R. Acharya, R. J. Martis, C. K. Chua, L. C. Min, E. Y. K. Ng, A.Laude, "Evolutionary algorithm based classifier parameter tuning for automatic diabetic retinopathy grading: a hybrid feature extraction approach," Knowl. Based Syst., vol. 39(0), 2013, pp. 9-22.

[12] J. J. Staal, M.D. Abranoff, M. Niemeijer, M.A. Viergener, B. van Ginneken, "Ridge based vessel segmentation in color images of the retina," IEEE Trans. Medical Imaging, Vol. 23, pp. 501-509, 2004.

[13] Mookiah, M.R.K., Acharya, U.R., Chua, C.K., Lim, C.M., Ng, E., Laude, "A..Computer-aided diagnosis of diabetic retinopathy: A review”. Comput Biol Med 2013;43(12):2136-2155.

[14] A Mendulkar, R Kale, A Agrawal, "A survey on efficient human fall detection system", International journal of scientific \& technology research, 2014.

[15] V Mal, AJ Agrawal, "Removing Flaming Problems from Social Networking Sites using Semi-Supervised Learning Approach", Proceedings of the Second International Conference on Information and Communication Technology for Competitive Strategies,2016. 